\title{
Deformation Profiles in Solid State Extrusion of High Density Polyethylene
}

\author{
Tetsuo Kanamoto, Anagnostis E. Zachariades, and Roger S. Porter \\ Polymer Science and Engineering Department, Materials Research Laboratory, \\ University of Massachusetts, Amherst, Massachusetts 01003, USA.
}

(Received July 24, 1978)

\begin{abstract}
The deformation mode on solid (crystalline) state extrusions for a series of high density polyethylenes (HDPE) has been investigated as a function of extrusion draw ratio (EDR) and molecular weight $\left(\bar{M}_{w}\right)$. The effects of extrusion temperature, rate, die entrance angle and lubrication have also been studied. The deformation flow profiles were observed by printing a lateral line pattern on the flat surface of the initially split billet and also by inserting colored HDPE filaments into split and unsplit billets perpendicular to the extrusion direction. It was found that the mode of deformation changed from a flat or even concave form through an undulant shape to a shear parabolic shape with increasing EDR for polyethylenes with $\bar{M}_{w}<150,000$. For higher- $\bar{M}_{w}$ polyethylenes, the flat or concave flow pattern observed at very low EDR changed to a W-shape pattern which has not been previously reported. The extrusion variables did not alter significantly these deformation patterns.
\end{abstract}

KEY WORDS Solid State Extrusion / High Density Polyethylene / Deformation Patterns / Extrusion Draw Ratio / Morphology /

There are many active studies on the solid state extrusion of semicrystalline polymers. In particular, the mode of deformation has attracted attention $^{1-5}$ since it aids the determination of the morphological changes that occur during extrusion and the resultant properties of the extruded films or filaments.

Imada and Takayanagi ${ }^{1}$ have proposed a possible mechanism for the solid state extrusion of high density polyethylene. They base their argument on a modified theory for the plastic deformation of metals. The calculated flow pattern for polymer extruded through a conical die with an entrance angle $60^{\circ}$ (in their definition taper angle $30^{\circ}$ ) was in reasonable agreement with the experimentally observed pattern. Utilizing these results, Kolbeck and Uhlman ${ }^{2}$ concluded that shear is the predominant deformation in the solid state extrusion of semicrystalline polymers. On the other hand, flat or even concave flow profiles have been observed recently at low extrusion draw ratios (EDR) by hydrostatic extrusion $^{3}$ and by the solid state coextrusion technique developed in our laboratory., ${ }^{4,5}$ More- over, our recent studies pertaining to the effects of molecular characteristics on extrusion behavior have revealed the formation of novel deformation profiles which change with EDR and molecular weight $\left(\bar{M}_{w}\right)$. In view of these results, we would like to report the mode of solid state deformation of semicrystalline polyethylene under a wide variety of extrusion conditions. The effects of extrusion temperature, die angle, and lubrication are also discussed.

\section{EXPERIMENTAL}

High density polyethylene billets of different molecular weight and source, see Table I, were compression molded to $0.95 \mathrm{~cm}$ in diameter in vacuo in a specially designed apparatus described elsewhere. ${ }^{4}$ The billets were split longitudinally into halves and imprinted with a lateral line pattern in order to measure the extent of draw and observe the deformation mode on extrusion. For the examination of deformation profiles perpendicular to the free surface of the split billet, colored polyethylene strands were inserted normal 
Table I. Characteristics of high-density polyethylenes

\begin{tabular}{lrrr}
\hline Polymer grade & $\bar{M}_{w} \times 10^{-3}$ & $\bar{M}_{w} / \bar{M}_{n}$ & $\begin{array}{c}\text { Melt } \\
\text { index }\end{array}$ \\
\hline Alathon 7050 & 57 & 3 & 17.5 \\
Alathon 7030 & 97 & 3.5 & 2.8 \\
Alathon 7026 & 147 & 4.5 & 1.0 \\
Marlex 6050 & $\leq 110$ & $7-13$ & 5.0 \\
Marlex 6003 & $=200$ & $7-13$ & 0.3 \\
Marlex 6002 & $\leq 250$ & $13-20$ & 0.2 \\
\hline
\end{tabular}

to the flat surface as shown in Figure 4. The two halves were assembled and press-fit into the reservoir of an Instron capillary rheometer maintained at the desired extrusion temperature. The split billets were extruded through conical brass dies having included entrance angles of $20^{\circ}$ and $60^{\circ}$ when the effect of die angle on the extrusion behavior was studied. To study the deformation mode as a function of the $\bar{M}_{w}$, split billets of different $\bar{M}_{w}$ were extruded at $120^{\circ} \mathrm{C}$ under a constant pressure of $0.23 \mathrm{GPa}$ through a die of EDR 25. The deformation mode was also examined as a function of the draw ratio. In this case, split billets of the same high density polyethylene were extruded through conical dies of the same entrance angle $\left(20^{\circ}\right)$ but different EDR and under different conditions as shown in Table II. Furthermore, the deformation mode was studied for selected polyethylenes as a function of the extrusion tem- perature. For this, split billets of the same polymer were extruded through a die of a draw ratio of 25 and under a constant pressure of $0.23 \mathrm{GPa}$. The deformation mode of unsplit billets was also examined by inserting colored polyethylene strands of the same HDPE as the billet to preformed billets normal to the extrusion direction. Extrusions were performed again through conical dies $\left(20^{\circ}\right)$ of EDR 12 and 25. In general, no lubricant was used and the billets, dies and rheometer barrel were washed thoroughly with acetone prior to each extrusion. In separate experiments the effect of silicone and teflon lubricant were studied.

\section{RESULTS AND DISCUSSION}

The effect of molecular weight on flow patterns is shown in Figure 1 for steady state extrusion at $0.23 \mathrm{GPa}, 120^{\circ} \mathrm{C}$ and extrusion draw ratio 25 . The characteristics of the high-density polyethylenes (HDPE) used, are shown in Table I. The extrudates exhibited a diminishing parabolic shear flow pattern indicated by the depth of the parabola, as the $\bar{M}_{w}$ was increased. The high molecular weight, Marlex 6003 and Marlex 6002 (not shown in Figure 1) exhibited clearly a novel W-shaped flow pattern. A tendency for such a pattern is also exhibited by samples Alathon 7026 and Marlex 6050. Such a complex deformation pat-

Table II. Extrusion data of solid state extruded HDPE

\begin{tabular}{|c|c|c|c|c|c|}
\hline \multirow[t]{2}{*}{ Polymer grade } & \multicolumn{4}{|c|}{ Extrusion conditions } & \multirow[t]{2}{*}{$\begin{array}{c}\text { Extrusion rate, } \\
\mathrm{cm} / \mathrm{min}\end{array}$} \\
\hline & EDR & $T,{ }^{\circ} \mathrm{C}$ & $P,(\mathrm{GPa})$ & Die angle, ${ }^{\circ}$ & \\
\hline \multirow[t]{5}{*}{ Alathon 7050} & 12 & 110 & 0.07 & 20 & \\
\hline & 25 & 120 & 0.16 & 20 & 4 \\
\hline & 25 & 90 & 0.23 & 20 & 0.14 \\
\hline & 36 & 120 & 0.23 & 20 & 1.5 \\
\hline & 25 & 120 & 0.23 & 60 & 0.43 \\
\hline Alathon 7030 & 25 & 120 & 0.23 & 20 & 1.0 \\
\hline Alathon 7026 & 25 & 120 & 0.23 & 20 & 0.15 \\
\hline Marlex 6050 & 25 & 120 & 0.23 & 20 & 0.8 \\
\hline \multirow[t]{5}{*}{ Marlex 6003} & 12 & 110 & 0.10 & 20 & \\
\hline & 25 & 120 & 0.23 & 20 & 0.06 \\
\hline & 25 & 132 & 0.23 & 20 & 0.44 \\
\hline & 25 & 135 & 0.23 & 20 & 0.80 \\
\hline & 25 & 120 & 0.23 & 60 & 0.015 \\
\hline Marlex 6002 & 25 & 120 & 0.23 & 20 & 0.05 \\
\hline
\end{tabular}




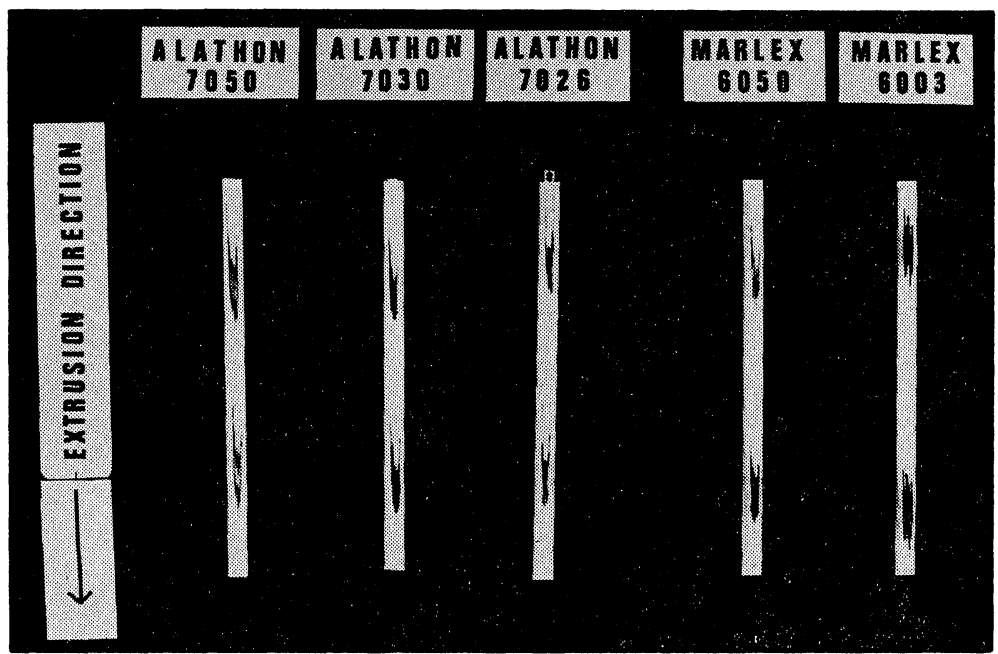

Figure 1. Deformation flow patterns at a constant EDR 25. Extrusion at $120^{\circ} \mathrm{C}$ and $0.23 \mathrm{GPa}$ pressure using no lubricant through a die of $20^{\circ}$ entrance angle. Flow pattern changes with polyethylene $\bar{M}_{w}$ (see Table I).

tern has not been reported previously for solid state deformation of semicrystalline polymers. This novel deformation flow may not only reflect the shear flow observed with the lower molecular weight polyethylenes but also the complex stress and strain field parameters in the extrusion die. Yield and flow stresses for HDPE under the high pressure ${ }^{6,7}$ strain and temperature of the extrusion condition are not well established parameters to allow a complete quantitative treatment. To investigate the nature of the novel deformation mechanism, we examined further the effects of several extrusion variables; these are $\bar{M}_{w}$, extrusion draw ratio (EDR), extrusion temperature, die angle, and lubricant. As the deformation proceeded in the conical part of the extrusion die, the flow patterns changed gradually as shown in Figure 2. The polyethylenes used were extruded at the same condition with those shown in Figure 1. The deformation patterns at very low EDR (the upper lines at the conical part) all appear as either concave or flat irrespective of the molecular weight. However, the difference in the flow patterns becomes distinct with increasing EDR, i.e., proceeding towards the tip of the conical die. Near the die exit (the lower line, higher EDR) the lower- $\bar{M}_{w}$ polyethylenes exhibited a shear parabolic flow profile whereas the high molecular weight polymer Marlex 6003 showed the novel
W-flow pattern.

In order to clarify the effects of EDR, two typical polyethylenes, Alathon 7050 and Marlex 6003 , were extruded through brass dies having nominal EDR 12, 25 and 36. The entrance angle of these dies was $20^{\circ}$. The maximum EDR calculated from the separation of flow lines was in excellent agreement with the nominal EDR defined by the ratio of the inlet to outlet cross sectional areas of the die. Figure 3 shows the deformation patterns of these extrudates observed at constant EDR. The extrusion rate of the high- $\bar{M}_{w}$ polyethylene Marlex 6003 was so slow that the maximum EDR practically obtainable was 25 under the extrusion conditions shown in Table II. As it was anticipated, the flow pattern changed from an approximately flat one at low EDR 12 to a typical shear flow (pattern) at the higher EDR 25 with the lower- $\bar{M}_{w}$ Alathon 7050. However, no such transformation occurred with the high- $\bar{M}_{w}$ polyethylene Marlex 6003, exhibiting the novel Wshaped profile even at the low EDR 12. The deformation profiles perpendicular to the interface of a split billet were obtained by inserting colored polyethylene strands perpendicular to the interface. The strands were cut along the free surface of the spit billet. The billet was extruded at $110^{\circ} \mathrm{C}$ through a conical die having an EDR 12 and entrance angle $20^{\circ}$. As shown in Figures 3 


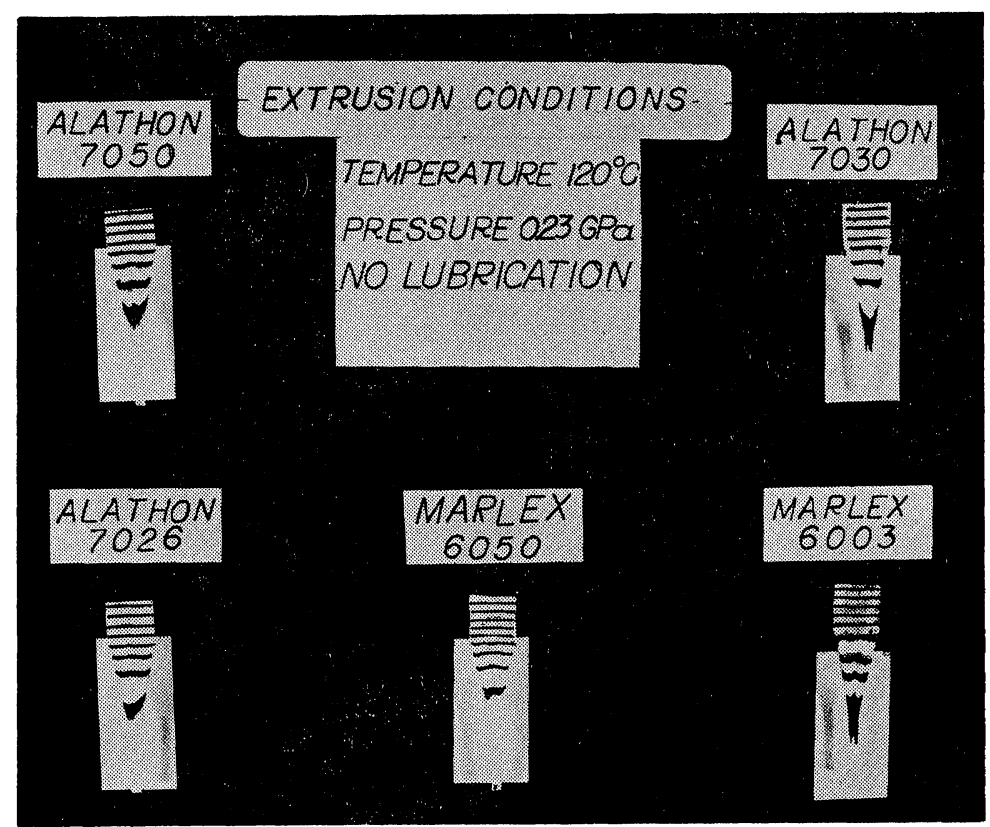

Figure 2. Deformation patterns in the conical entrance zone of the extrusion die. The extrusion conditions are the same as those shown in Figure 1.

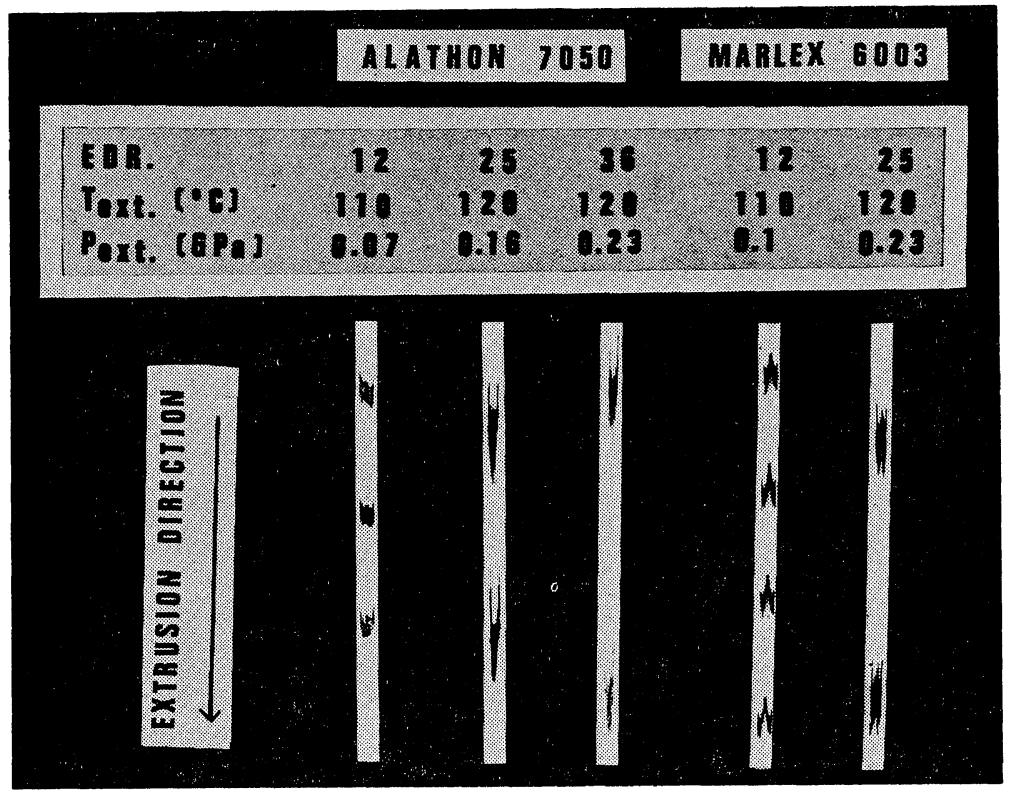

Figure 3. Effects of EDR on flow profiles for the lowest- $\bar{M}_{w}$ (Alathon 7050) and high- $\bar{M}_{w}$ (Marlex 6003) polyethylenes. Extrusion conditions are as indicated.

and 4 , the flow profiles on the interface of the two half billets (Figure 3) are identical with those observed perpendicularly to the interface (Figure 4). Such an observation indicates that the characteristic flow patterns are uniformly distributed and cylindrically symmetrical throughout the extrudates. A preliminary study of the deformation profiles of unsplit billets of Alathon 


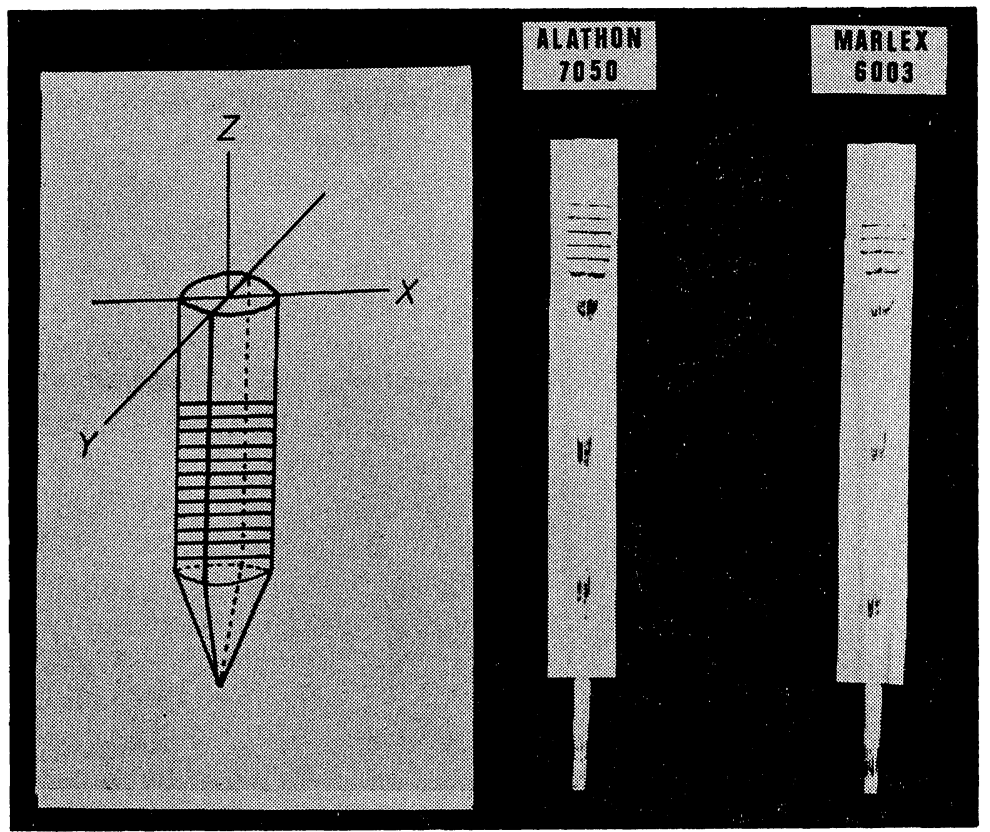

Figure 4. Deformation pattern in a plane perpendicular to the flat surface of the split billet. The patterns are similar with those observed on the flat surface of split billets in Figure 3 . The EDR and temperature were 12 and $110^{\circ} \mathrm{C}$. The extrusion pressure was $0.10 \mathrm{GPa}$ for Marlex 6003 and $0.07 \mathrm{GPa}$ for Alathon 7050.

7050 and Marlex 6003 indicated that the profiles become indistinguishable from those observed with split billets as the EDR becomes high (EDR $>12)$ under the same extrusion conditions. This fact indicates that the $\mathrm{W}$ flow pattern is characteristic of high- $\bar{M}_{w}$ HDPE irrespective of the extrusion technique.

As evidenced by the large changes in the extrusion rate shown in Table II, the extrusion temperature greatly affects the yield and flow stress under high pressure and strain. Nevertheless, although the shear parabolic flow profile increased slightly with the extrusion temperature, there was a major difference in flow pattern between high and low molecular weight polyethylenes (Figure 5).

The stress field during deformation at the conical part of the die may be affected by the die angle and friction coefficient between the polymer and die wall. In the case of melt extrusion, flow patterns change from shear to extensional, with decreasing die entrance angle. ${ }^{8}$

Flow line profiles of the Alathon 7050 and Marlex 6003 samples extruded through a die having an entrance angle $60^{\circ}$ and an extrusion draw

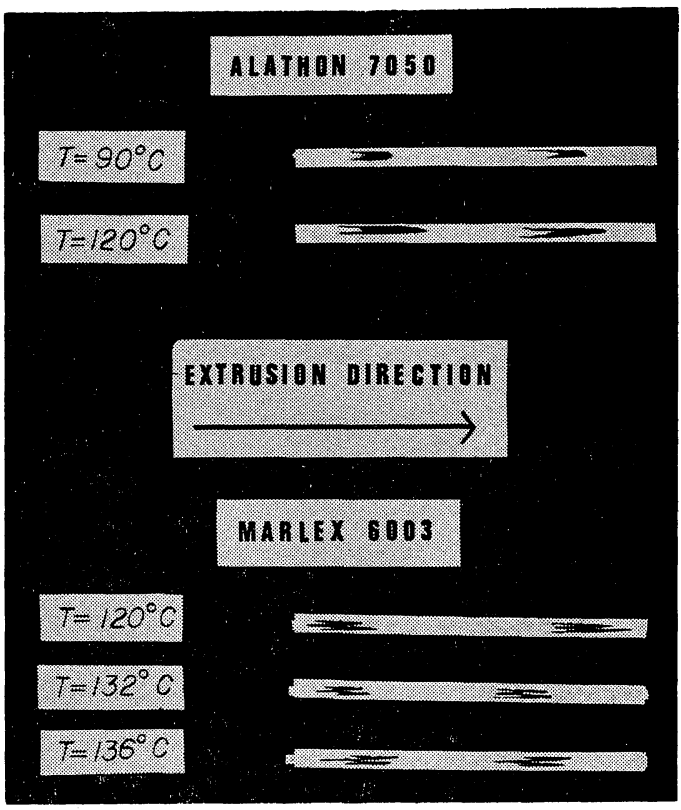

Figure 5. Deformation pattern changes with extrusion temperature for low (Alathon 7050) and high (Marlex 6003)- $\bar{M}_{w}$ polyethylenes. 


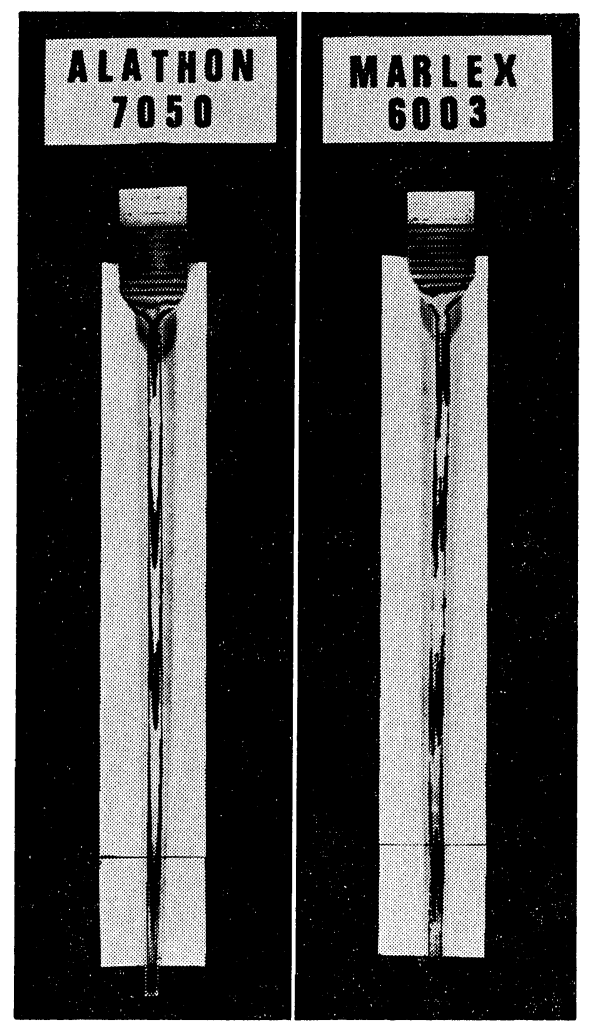

Figure 6. Deformation patterns obtained by using a die having entrance angle $60^{\circ}$, EDR 25 and extrusion temperatures and pressures of $120^{\circ} \mathrm{C}$ and $0.23 \mathrm{GPa}$; the same as for extrusion using a die of entrance angle $20^{\circ}$ (Figure 1).

ratio of 25 are depicted in Figure 6. The extrusion conditions were the same with those reported previously for extrusion through a die with an entrance angle $20^{\circ}$, as illustrated in Figure 1 . In general, the flow profiles resemble those obtained previously for steady state extrusion through a die of entrance angle $20^{\circ}$, i.e., a shear parabolic and the characteristic $\mathrm{W}$ profile for Alathon 7050 and Marlex 6003 samples respectively. A closer examination of the lower draw ratio regime in the die shows that the die angle influences the flow profile and visual changes may be observed at the surface regions near the die wall. A flat flow pattern may be assumed which changes to the parabolic shear or W-shaped at the tip of die. Furthermore, the die angle affects the extrusion rate which decreases significantly with the die angle as shown in Table II. The effect of lubrica- tion on flow patterns was examined by coating the die and barrel wall with silicon oil and poly (tetrafluoroethylene) in separate experiments. No apparent effect was detected on the flow patterns. The reproducibility of flow patterns was excellent at low extrusion rates. However, a slight distortion or asymmetry of flow patterns sometimes occurred at high extrusion rates. In an extreme case, when stick-slip occurred during extrusion, the patterns were significantly distorted.

It appears that the flow lines transform from an approximately flat profile through an undulant to a shear parabolic profile with extrusion draw ratio for polyethylenes of $\bar{M}_{w}<150,000$. Although the extrusion temperature considerably affects the depth of the shear parabolic well or the length of the limbs of the $\mathrm{W}$ profile, it nevertheless leaves the overall profile unaltered. The extrusion die angle effect is analogous. The structure and properties of oriented semicrystalline polymers have been usually discussed as a function of the draw ratio, i.e., the macroscopic extent of deformation imposed on the sample and the deformation conditions. However, the deformation modes may also play an important role in determining the morphology variation ${ }^{9,10}$ along and/or across the deformed sample and improving the resultant properties.

The W pattern can be thought of as being composed of a shear component-the limbs of the $W$ profile-and a chevron component_-connecting the limbs of the profile. Such a pattern suggests the operation of complex deformation modes. Shear is one component mode. Other component(s) should result in the chevron region of the profile which becomes profound as the $\bar{M}_{w}$ increases. Some of the reason giving rise to this deformation tendency are heat effects and chain rupture arising from surface friction and shearing, and morphological variation across the preformed billet. However, there is no detectable temperature elevation during the solid (crystalline) state extrusion of $\mathrm{HDPE}^{2}$ under the conditions similar to those employed in our experiments. Furthermore, chain rupture ${ }^{11}$ under our extrusion conditions does not appreciably affect the deformation flow. On the other hand, thermal analysis and optical microscopy showed no morphological variations across the preformed billets. Imada suggested, on the basis of slip line analysis, ${ }^{12}$ that when the 
frictional coefficient is large in comparison with the yield stress or, alternatively, when strain hardening is not appreciable, a W-deformation mode may be possible. However, stress-strain relationships under high pressure and temperature for HDPE are not well known. Furthermore, such relationships should be rate dependent in the case of crystalline state extrusion as manifested by the logarithmic increase in extrusion rate with applied pressure. ${ }^{13-15}$ Thus a quantitative analysis by continuum mechanics is difficult at the present time. The nature of the flow transformation during extrusion and the W-shaped profile are currently under investigation.

\section{CONCLUSION}

Results indicate that the mode of deformation during the solid state extrusion of a series of HDPE samples of different molecular weight is complex, depending on a number of variables. The deformation profile transforms from a flat (linear) to a shear parabolic with extrusion draw ratio for PE of $\bar{M}_{w}<150,000$. Polyethylenes of $\bar{M}_{w} \geq 200,000$ show a novel W-shaped profile which may be attributed to the complex stress and strain field in the extrusion die, implying a shear plus additional deformation mechanism(s). Although the extrusion temperature and die angle affect significantly the extrusion rate, they do not alter the characteristic shape of the flow profiles.

Acknowledgment. The authors express their appreciation to Dr. T. Shimada for his valuable comments.

\section{REFERENCES}

1. K. Imada and M. Takayanagi, Int. J. Polym. Mater., 2, 89 (1973).

2. A. G. Kolbeck and D. R. Uhlmann, J. Polym. Sci., Polym. Phys. Ed., 15, 27 (1977).

3. N. Inoue and T. Nakayama, Technocrat., 10, 23 (1977).

4. P. D. Griswold, A. E. Zachariades, and R. S. Porter, presented, Stress-Induced Crystallization Symposium, Midland Macromolecular Institute, Midland, MI, August 1977.

5. T. Shimada, A. E. Zachariades, W. T. Mead, and R. S. Porter, accepted, J. Crystal Growth.

6. K. D. Pae and D. R. Mears, J. Polym. Sci., Part $B, 6,269$ (1968).

7. K. D. Pae, D. R. Mears, and J. A. Sauer, $J$. Polym. Sci., Part B, 6, 773 (1968).

8. J. W. Hill and J. A. Cuculo, J. Macromol. Sci.Rev. Macromol. Chem. C14(1), 107 (1976).

9. R. G. Crystal and J. H. Southern, J. Polym. Sci., Part A-2, 9, 1641 (1971).

10. C. J. Farrell and A. Keller, J. Mater. Sci., 12, 966 (1977).

11. W. T. Mead and R. S. Porter, to be published.

12. K. Imada, private communication.

13. C. J. Farrell and A. Keller, J. Mater. Sci., 12, 996 (1977).

14. W. T. Mead and R. S. Porter, accepted by $J$. Polym. Sci., Part C.

15. T. Kanamoto, A. E. Zachariades, and R.S. Porter, presented at APS Meeting, Washington, D. C., March, 1978, to be published. 